\title{
Quels facteurs géographiques prendre en compte pour mieux gérer la grande faune?
}

\author{
Yves Poinsot \\ Géographe, Université de Pau et des Pays de l'Adour, IRSAM, UMR5603 SET, 64000 Pau, France
}

\begin{abstract}
Cet article pose les premiers jalons d'une nouvelle perspective gestionnaire de la faune sauvage qui se définirait non plus en fonction de l'espèce elle-même, mais des territoires dans lesquels la faune évolue. L'auteur montre en quoi le statut des espèces animales, qu'elles soient considérées comme nuisibles ou non, est insuffisant pour en assurer la gestion, car il ignore les propriétés fonctionnelles du territoire avec lesquelles l'animal compose. Cette perspective, même encore lointaine et mise en débat à la faveur de la création des schémas départementaux de gestion cynégétique de la loi sur la chasse, interroge les cadres d'action publique dans leur capacité à promouvoir une différenciation des objectifs et des modalités de gestion selon les territoires.
\end{abstract}

La Rédaction

\section{Mots-clés :}

milieu ; géographie ; gestion ; loup ; sanglier

\section{Keywords:} environment; geography; management; wolf; wild boar
Résumé - Gérer la grande faune, pour maîtriser les proliférations ou protéger les espèces, exige de prendre en compte la diversité du milieu géographique. On le définit par trois facteurs ayant du sens à la fois dans la dimension naturelle et sociale : la densité, l'accessibilité et la forme. Leur mobilisation, seuls ou en combinaisons, éclaire d'un jour nouveau la gestion des grands ongulés et de leurs prédateurs. Pour le sanglier, densités des animaux comme des hommes, parfois combinées à des effets de formes ou d'accessibilité, expliquent les modalités de sa gestion, différenciée selon les régions. Pour le loup, sa répartition dépend de facteurs trophiques, concurrentiels intraspécifiques, voire topographiques, mais aussi sociaux. Ses lieux de présence en Espagne résultent de combinaisons mêlant les densités des hommes, des proies naturelles et du bétail, ainsi que des relations unissant chasseurs et éleveurs.

\begin{abstract}
A focus on the geographical factors allowing to better manage large wildlife. To manage large wildlife, control proliferations or protect species, one needs to work on extensive territories and take the diversity of the geographical environment into account. This is defined by three main factors that are meaningful from both natural and social standpoints, i.e., density, accessibility and shape. Alone or combined, they offer new perspectives on the management of wild boar and wolf. Regarding wild boars, animal as well as human density, sometimes linked to the effects of shape or accessibility, accounts for the diversity of management methods applied in the different French régions. As for wolves, their perception of the environment as either friendly or non-friendly depends both on natural (trophic, intraspecific competition, topographic) factors and social factors (breeding conditions, legal status). Under the pressure of control culling adjusted to local situations, feelings change with increasing densities. The study of Spanish situations shows how wolf distribution is due to a combination of human density, natural preys and cattle farming and also to the relationships between hunters and livestock farmers. To conclude, we propose to include the density ratio between farmers and hunters in management parameters. We argue that the legal status of species should change when densities increase based on a département spatial scale.
\end{abstract}

\section{Introduction}

La conquête des Alpes par le loup a fait envisager la délimitation de zones «refuges», «favorables» et «inaptes» (Bobbé, 2002b). L'accroissement inégal des dégâts de sangliers aux maïs landais a conduit la fédération des chasseurs à différencier les montants du timbre "grand gibier» au sein même de l'espace départemental.

Auteur correspondant : yves.poinsot@univ-pau.fr
La loi sur la chasse de 2000, en instituant des schémas départementaux de gestion cynégétique, a suscité un débat sur les limites territoriales des unités de gestion : réutiliser celles des pays ou adopter celles des grands biotopes (Arnauduc, 2008). Ces trois situations révèlent que la gestion de la grande faune, pour la protéger ou maîtriser ses proliférations, suscite une réflexion géographique.

Pour la conduire, gestionnaires d'espaces naturels protégés ou de territoires cynégétiques renseignent 
souvent d'informations relatives aux dimensions, aux formes (topographiques ou des zonages), voire à l'accessibilité, les couches géolocalisées des systèmes d'information géographique. Par une superposition maîtrisée de l'information, l'outil devrait ainsi révéler en quoi l'espace participe à l'explication des problèmes. Mais les recherches géographiques récentes n'apportent que peu de concepts utilisables dans ces perspectives. Dans le champ géohistorique, une contribution érudite (Planhol, 2004) complète les productions d'historiens (Delort, 1984) en analysant la diversité des représentations suivant les espèces, leur rareté, ou encore le niveau de développement des sociétés. La géographie des acteurs explore de son côté les champs géopolitique (Benhammou, 2007) et socioculturel (Mounet, 2007), dans le prolongement de travaux explicitement sociologiques (Mauz, 2005). Étoffant la connaissance des représentations et des conflits d'acteurs, ces travaux parlent cependant plus du rapport des hommes au territoire, au travers des relations à l'animal, que de celui des animaux à leur milieu géographique, dans sa diversité naturelle et sociale.

Or, parce que la grande faune est mobile, à l'échelle des navettes d'un individu dans son domaine vital comme à celle de la colonisation d'un territoire par une population (le loup dans l'Hexagone, par exemple), penser le milieu animal sans le spatialiser n'est pas tenable d'un point de vue gestionnaire. Dans ce cadre, la gamme des variables géographiques à prendre en compte inclut des déterminants valides à la fois dans les champs naturels et sociaux dont nous proposons, dans cet article ${ }^{1}$, de discuter la nature et les combinaisons. Nous considérons pour cela que, dans l'Ancien Monde densément humanisé, le milieu géographique où se reproduit l'animal est la résultante d'un façonnement social ancien, en évolution constante, qui concerne les couverts végétaux (agricoles et forestiers), les statuts réglementaires (des zones protégées et des zones chassables), voire des représentations sociales (des déprédations acceptables - sur la forêt, par exemple - et d'autres non - sur la vigne, notamment). Dans ce contexte, la qualité d'accueil d'un milieu géographique pour une harde d'ongulés ou une meute de loups repose sur l'existence, dans un périmètre propre à chaque espèce, $d$ 'une combinaison de lieux reliés par des itinéraires de déplacement qui font sa qualité (tranquillité des refuges, abondance et régularité de l'alimentation, facilité des navettes) : on est là dans l'objet qu'étudie l'écologie du paysage (Burel et Baudry, 1999).

Dans ce modèle répartissant les rôles de façon claire, les uns façonnant un milieu dans lequel s'inscrivent les

\footnotetext{
${ }^{1}$ Cet article est issu d'un programme de recherche intitulé : «Protection, prédation, production: contribution géographique à la gestion durable de la faune sauvage en Aquitaine », financé par le comité consultatif régional pour la recherche et le développement technologique (CCRRDT) d'Aquitaine (2008-2010).
}

autres, les situations de coexistence pacifique (l'existence viable de la grande faune sans conflits liés aux déprédations) ne sont qu'assez rares. Or, mieux comprendre pourquoi, dans telle partie du bocage normand ou des plateaux jurassiens, la gestion de la grande faune, pour la protéger ou la réguler, paraît plus facile que dans telle autre n'est pas inutile. Si les déterminants sociétaux sont essentiels (l'acceptabilité territoriale des espaces naturels protégés n'est pas la même partout, les conditions d'entente entre les chasseurs sont inégalement réparties...), l'hypothèse centrale de cet article est que trois variables géographiques en interrelations s'imposent aux hommes comme aux animaux et participent de manière déterminante aux conditions de cette coexistence, apaisée ou conflictuelle.

La première variable concerne les densités, dont le niveau élevé chez les uns tend à abaisser l'importance chez les autres, par un jeu de vases quasi communicants. La seconde relève des formes, qu'elles concernent les couverts végétaux façonnés (la mosaïque d'un bocage, les grandes masses forestières des Landes), les contours et la taille d'une aire protégée (massive ou allongée, elle ne fournira pas le même refuge aux espèces suivant la taille de leurs domaines vitaux) ou la topographie (dont les contraintes éloignent les hommes et attirent la faune). La troisième concerne l'accessibilité : lorsqu'elle est bonne d'un point de vue humain, elle est source de dérangements pour les animaux (par les pistes forestières trop nombreuses, etc.), voire permet leurs régulations même dans les remises reculées ; lorsqu'elle est déficiente pour les hommes (par une réglementation restrictive ou simplement une topographie contraignante), elle favorise la tranquillité de la faune. À l'inverse, quand on veut réduire les déprédations animales, on peut installer des clôtures protectrices.

Si on distingue ces trois variables pour la commodité de l'exposé, leurs conditions d'intervention sont interdépendantes (la possibilité d'enclore liée à la forme de la parcelle) et jouent souvent de concert (c'est quand les densités montent qu'on cherche à restreindre l'accessibilité, par exemple). C'est donc pour éclaircir leurs conditions d'intervention qu'une recherche a été menée, portant sur l'examen, pendant quatre ans, des méthodes de contrôle des densités de grands ongulés - sangliers principalement - et de protection-régulation du loup dans et autour de parcs et réserves naturels français et espagnols : notamment, en France, Pyrénées occidentales, Cévennes, Mercantour, Caroux-Espinouse, Vercors ; en Espagne, Somiedo, Picos de Europa, Aigües Tortes et La Culebra $(\text { León })^{2}$. Elle a mis en lumière des règles de répartition de

2 L'information mobilisée ici résulte d'entretiens semidirectifs de 1 à 3 heures (voire des journées entières par la participation à des opérations de comptage) auprès des gestionnaires de réserve et des chasseurs et/ou éleveurs de la périphérie. La collecte d'informations n'a jamais porté sur moins 
la faune sauvage qui ne prétendent pas tout expliquer par la seule combinaison des trois variables, mais les considèrent néanmoins essentielles à la compréhension des relations géographiques liant le sauvage et l'humain.

Dans ce cadre problématique, nous nous attachons d'abord à préciser la nature de ces variables et celle de leurs relations. Nous discutons ensuite la façon dont cette grille de lecture permet de mieux comprendre succès et échecs dans la gestion du sanglier, choisi parmi les ongulés pour l'importance des dégâts qu'il commet et la vigueur de son taux de reproduction. Dans la troisième partie, nous tentons d'éclairer les incidences de ces déterminants sur l'inscription géographique du loup, insistant sur les effets des périmètres de sa protection et les conséquences de l'expansion de l'espèce sur ses rapports à l'élevage.

\section{Trois variables géographiques, conditions de l'équilibre gestionnaire}

Les densités humaines en Europe sont aujourd'hui, dans leur grandes masses urbaines, commandées par des facteurs parmi lesquels la nature pèse bien peu. Dans les campagnes aussi, l'histoire et les conditions techniques engendrent de fortes densités en des milieux peu fertiles (les campagnes bretonnes) et des densités faibles en des greniers agricoles (la Beauce). Si la nature n'explique donc pas tout, elle intervient encore largement pour justifier le peuplement lâche des régions montagneuses, les contraintes climatiques et l'insuffisance des moyens d'accès mécanisés interdisant une occupation agricole dense. Dans ce contexte, et sans y regarder pour l'instant de trop près, on constate que les grands ongulés sont plus rares dans les campagnes peuplées, bretonnes ou bordelaises, que dans le Massif central ou les Landes (Poinsot, 2008). Cela tient certes aux propriétés des couverts végétaux (les remises forestières y sont rares), mais aussi à l'inacceptabilité sociale du dégât : les hommes ne sont nombreux $q^{\prime}$ 'en raison de la forte valeur que produit chaque hectare, toute déprédation serait alors économiquement très coûteuse ; la régulation maintient donc la grande faune à des niveaux très bas. À l'inverse, quand les hommes se raréfient, l'élevage extensif ou la sylviculture deviennent la norme. Les revenus à l'hectare étant moindres, des déprédations modérées sont moins graves; par conséquent, à la fois parce que les couverts végétaux s'y prêtent et que l'acceptabilité de la déprédation s'améliore, les densités de la faune peuvent s'accroître. Elles peuvent atteindre des records lorsqu'une bonne productivité du milieu se conjugue à des densités humaines basses - les

de 6 à 10 entretiens par réserve, voire a dépassé la vingtaine pour les parcs des Cévennes, des Pyrénées occidentales et du Mercantour. Au total, plus de 150 personnes ont été rencontrées.
Cévennes constituent à cet égard un archétype sur lequel on reviendra.

À ces situations bien tranchées, s'opposent des situations intergrades où des densités animales fortes voisinent parfois avec des couverts végétaux de valeur économique élevée. C'est le cas quand des vignobles jouxtent des bosquets au sein d'un bocage, dans le Sud-Ouest, par exemple. On peut alors, pour des montants certes élevés, mais que couvrent les forts revenus à l'hectare, ceindre les parcelles de hauts grillages qui interdisent l'accès des chevreuils, notamment. Mais il faut que la forme des parcelles soit la plus massive possible, tout étirement dans la longueur augmentant le rapport périmètre/surface et donc le coût relatif de la clôture. À une variable «densité» facteur de dégâts, on répond donc ici par une réduction de l'accessibilité (la clôture) pour peu que la forme le permette.

Mais, si les surfaces à protéger sont trop importantes ou les revenus à l'hectare insuffisants, il faut alors agir sur les effectifs de déprédateurs : c'est le rôle du plan de chasse que de maintenir un "équilibre agrosylvocynégétique » acceptable par tous. Ce mode de gestion des campagnes banales se heurte souvent à des contraintes d'accessibilité ou de formes. En zone alpestre, en effet, les difficultés de régulation ou les possibilités de protection, suivant le regard porté sur les espèces, doivent leur origine à une accessibilité restreinte par la pente. Lorsque de longs versants sans accès sylvopastoraux imposent de longues marches, la régulation des grands cervidés fait problème, les chasseurs ne pouvant ramener les bêtes abattues, trop lourdes, jusqu'aux routes. Pour le loup en phase d'expansion, ces versants d'accessibilité pédestre difficile constituent des remises de choix, expliquant pour partie leurs zones de conquête préférentielles dans l'arc alpin.

Dans le cas des aires protégées, leur forme inadaptée peut restreindre leur efficacité pour la protection des grands animaux. La figure allongée au long de la frontière espagnole du Parc national des Pyrénées occidentales ne lui confère ainsi qu'une profondeur minime, souvent quelques kilomètres seulement, inapte à couvrir le domaine vital d'un ours adulte. Le débordement de l'espèce hors de l'aire protégée est donc inéluctable, affaiblissant une protection que des parcs de surface voisine mais de formes plus massives assureraient mieux. Si la conjugaison d'une forme adaptée et d'une réduction de l'accessibilité des chasseurs constitue un facteur de protection d'une espèce, elle engendre aussi un effet "parapluie » souvent préoccupant. Des grands ongulés percevant bien l'absence de prédation cynégétique que procure l'abri viennent ainsi s'y réfugier et, par leurs densités accrues, engendrent des pressions trophiques sur les couverts végétaux, voire sur certaines espèces animales, mettant en péril leur reproduction. Le façonnement des couverts n'est pas, dans ce cas, le seul apanage des sociétés; cela 
confirme ce que le retour du loup à Yellowstone avait enseigné sur l'évolution différencié des paysages végétaux suivant le degré de crainte inégalement réparti qu'ils inspirent aux proies potentielles (Ripple et Beschta, 2004).

Ces premiers éléments soulignent qu'accessibilité, forme et densité constituent, pour les sociétés comme pour les animaux, des facteurs de répartition relevant d'une topologie pour la première, d'une géométrie pour la seconde et d'une écologie animale et humaine pour la troisième, celle du rapport entre population et ressources $^{3}$. Ils montrent aussi que, si ces trois variables jouent individuellement un rôle majeur, c'est de leurs combinaisons que résultent souvent les propriétés du milieu géographique qui permettent le développement ou le recul des espèces. Leur diversité pouvant a priori être infinie, l'étude de cas précis empruntés aux sphères du sanglier puis du loup permet de saisir en quoi, si les principes sont communs, la combinatoire change des grands ongulés aux grands carnivores. Les uns, densément présents dans tout l'Hexagone, commettent des dommages aux ressources agricoles mais peuvent constituer des ressources cynégétiques, tandis que les autres, seulement perçus comme des prédateurs en phase de colonisation, se prémunissent de la menace régulatrice par une diffusion préférable à une hausse des densités.

\section{Le sanglier dans son milieu : conditions géographiques de son alimentation, conditions cynégétiques de sa régulation}

On mentionne le sanglier ${ }^{4}$ pour les dégâts qu'il occasionne aux cultures ${ }^{5}$ ou les collisions routières qu'il engendre ${ }^{6}$. Deux types de remèdes non exclusifs sont alors employés. Les premiers, jouant sur l'accessibilité, consistent en des clôtures qui peuvent être lourdes,

\footnotetext{
${ }^{3}$ Il prend souvent la forme de densités relatives, lorsque deux espèces se concurrencent pour l'accès à une même ressource ou que deux groupes sociaux envisagent des modes de valorisation du milieu incompatibles. Pour ces derniers, la rivalité se règle alors soit par le biais d'un marché (les plus puissants prennent le dessus, par la maîtrise du foncier notamment), soit par des élections (c'est alors l'avis des plus nombreux qui s'applique).

4 Baubet (1998) propose des références précises sur l'écologie de l'espèce, Klein et al. (2008) sur les problématiques de sa gestion et Pelosse (1993) sur les pratiques cynégétiques le concernant. Le Bulletin mensuel de l'Office national de la chasse puis Gibier faune sauvage ont publié de nombreux articles sur la gestion et le comportement territorial de l'animal.

5 Sur 22 à 23 millions d'euros de dégâts annuels aux cultures qu'indemnisent les fédérations de chasse, le sanglier représente $83 \%$ des montants (source : Fédération national des chasseurs).

6 En 2008, 42471 dossiers d'indemnisation «faune sauvage » ont été déposés auprès du Fonds de garantie automobile (FGA) pour un montant indemnisé de 21 millions d'euros : $40 \%$ concernaient le sanglier (source : FGA).
}

comme en bordure d'autoroute, mais sont plus fréquemment légères. Deux fils électriques disposés à 20 et 40 centimètres du sol constituent alors une «clôture à sangliers ». Les seconds, jouant sur la densité, consistent à maintenir une pression cynégétique suffisante pour que, la population régressant, les dégâts diminuent. Dans certains cas, leur mise en œuvre fait pourtant problème.

\section{Aux faibles densités humaines : maîtriser les proliférations pour préserver l'agriculture}

En raison des faibles densités humaines dans les Landes, les parcelles agricoles et forestières y atteignent plusieurs centaines d'hectares. De vastes îlots de maïs jouxtant ainsi de grands massifs boisés, les clôtures à sanglier sont la norme. Leur efficacité devient pourtant discutable quand les parcelles clôturées sont trop vastes et la densité des animaux trop élevée. Quand le maïs est haut, les sangliers affamés dans une pinède peu nourricière franchissent en effet la clôture sans que le cultivateur ne le décèle (la surveillance étroite d'un tel linéaire est difficile) et s'installent dans la parcelle plusieurs jours durant à l'abri des regards. L'importance des dégâts n'est alors mesurée qu'au moment de la récolte, et les montants sont élevés ${ }^{7}$. Il faut donc réduire les populations; mais comment définir une densité optimale dans un milieu théoriquement pauvre - la forêt de pins maritimes -, irrégulièrement « enrichi » par des accès incontrôlés à du maïs interdit mais tellement nourrissant qu'il détermine largement le taux de reproduction?

En Ardèche, la prolifération de l'espèce met en péril la survie agricole. L'espace-milieu présente des caractéristiques opposées à celles des Landes : la topographie est accidentée et le couvert forestier nutritif (châtaigniers et chênes verts); du point de vue social, le recul agricole est tellement accentué que les rares survivants - un ou deux par commune - se trouvent aujourd'hui minoritaires sur les plans spatial (des îlots de culture dans un océan forestier, $c f$. Photo) et politique (1 à 2 agriculteurs par commune quand les chasseurs sont entre 20 et 30). Dans ces campagnes où les niveaux de vie moyens sont modestes (peu de cadres, de nombreux retraités agricoles), la multiplication des sangliers est perçue comme une ressource : 20000 animaux sont abattus chaque année dans le département de l'Ardèche ${ }^{8}, 5000$ dans le seul Parc national des Cévennes ${ }^{9}$. Certaines «équipes à sangliers » n'excédant pas 10 à 15 chasseurs abattent 200 à 300 animaux par an, soit 10 à 20 par participant. On raisonne donc chambres froides et non plus congélateurs et les compléments de ressources deviennent significatifs. Convaincre

\footnotetext{
7591693 euros pour le département en 2008 (source : FDC des Landes).

8 Source: FDC de l'Ardèche.

9 Source: service cynégétique du Parc national des Cévennes.
} 


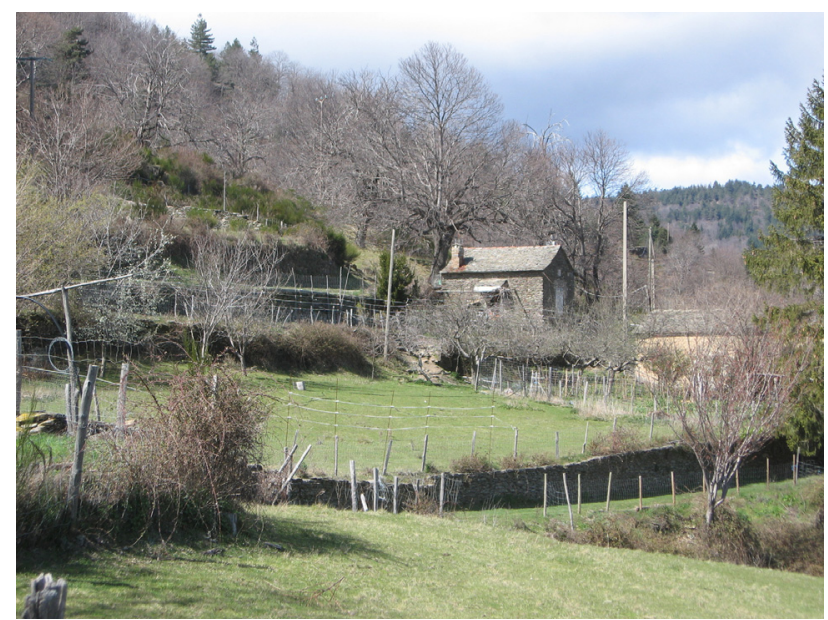

Photo. Le milieu géographique cévenol.

Le substrat schisteux qu'incise activement le réseau hydrographique engendre un système de pentes qui rend la mécanisation difficile, mais assure une production abondante de châtaignes. Le recul agricole est tel que les rares habitants doivent « s'enclore » dans un périmètre de grillages ou de clôtures électrifiées pour préserver potagers et vergers des déprédations du sanglier et du cerf. (Photo : Y. Poinsot.)

ces résidents (principaux ou secondaires), qui sont aussi électeurs, de réduire les populations de suidés pour protéger une activité agricole devenue minoritaire apparaît difficile. On rejoint ici des situations de l'Espagne intérieure où certains villages, identifiés depuis des siècles comme vivant d'une chasse autrefois autoconsommée, vivent aujourd'hui de l'accueil de chasseurs citadins venus faire des tableaux enviés (Lopez Ontiveros, 1991). Il s'est instauré dans les deux cas un rapport au milieu propre aux régions de basse densité où la production sylvicole, pour des raisons bioclimatiques, n'est que médiocre et où la faune sauvage devient une ressource majeure.

\section{Densités animales et structures sociales en périphérie des réserves}

Si les deux situations relèvent de campagnes banales où les facteurs «taille et morphologie des parcelles » et « rendement alimentaire du milieu forestier " s'avèrent déterminants, les difficultés se multiplient dans les espaces protégés. Les proliférations que favorise ce statut engendrent des auréoles de dégâts, et donc de "surchasse " dans les périphéries proches, mais aussi des atteintes aux espèces protégées au cœur du sanctuaire. Dans la Réserve nationale de chasse et de faune sauvage d'Arjuzanx, la reproduction des grues cendrées est menacée, alors que, dans les parcs nationaux des Pyrénées ou des Cévennes, c'est la survie du grand tétras qui est en jeu ${ }^{10}$. Lorsque l'ampleur du phénomène est mineure, les tirs de régulation qu'opèrent les agents de la réserve suffisent parfois. Leur nombre étant restreint, le recours à des tiers devient courant, bien que leur origine fasse débat.

Les chasseurs locaux, connaisseurs du terrain, répugnent à réguler au sein d'une réserve, dont ils attendent au contraire qu'elle serve de " réservoir » pour les secteurs chassables du pourtour. Si l'on veut recourir à des «extérieurs ", comment les encadrer sans mobiliser des agents? Dans les zones interdites à la chasse du Parc national des Cévennes, les agents du parc ou de l'Office national des forêts (ONF) ont délimité des sous-zones de 200 à 400 hectares au sein desquelles ils procèdent à un contrôle étroit de la pratique par une délivrance, jour par jour et lieu par lieu, de bracelets aux tireurs; pour les amateurs ne connaissant pas le terrain, un accompagnement payant peut même être assuré par l'ONF. C'est donc par la création de formes adaptées qu'on facilite le contrôle.

Dans les territoires périurbains, la régulation se heurte à deux catégories de contraintes. L'opposition philosophique de nombreux résidents à la pratique cynégétique constitue un obstacle majeur. Pourtant, formes et densités du mitage pavillonnaire, auxquelles s'ajoute le déclassement des territoires de chasse dans un rayon de 150 mètres autour des habitations, créent des réserves de fait : l'animal accède aux zones habitées sans que le régulateur puisse le suivre (Waguet et Charlez-Coursault, 1991). La multiplication des accidents routiers, voire des dégâts aux jardins ou aux piscines, impose l'organisation de battues administratives sous l'égide des lieutenants de louveterie, mais les agents de la force publique (ONF ou ONCFS [Office national de la chasse et de la faune sauvage]) sont trop peu nombreux : tirs nocturnes et parfois piégeage les occupent déjà dans les situations les plus critiques. Les battues par poussées silencieuses (sans chiens) sont donc les plus pratiquées. Mais qui mobiliser et comment? Les chasseurs locaux sont réticents (on leur demande ici d'éliminer une ressource qu'ils protègent par ailleurs), les riverains aussi; la rencontre d'un homme armé en tenue de camouflage aux limites du jardin accroît l'aversion. Les autorités peinent donc à rassembler des effectifs appropriés à la forme des territoires de chasse, facteur-clé d'une imperméabilisation suffisante de la ligne de tirs (Saldaqui, 2008; Poinsot et Saldaqui, 2009). Si, faute de rabatteurs et de tireurs en

\footnotetext{
10 Outre les témoignages de gardes des parcs nationaux concernés, Saniga (2002) confirme un phénomène qui s'inscrit dans les rapports liant les densités des espèces animales entre elles. Ces densités dépendent soit des relations prédateursproies (Jobin et al., 2000), soit des concurrences pour l'occupation d'une niche écologique donnée (voir, pour le coyote et le loup, Gompper, 2002). Les questions des espèces invasives comme des réintroductions relèvent de cette problématique.
} 
nombre suffisant, seulement $20 \%$ des populations excédentaires sont abattues chaque fois, il faudra répéter l'opération, ce qui multiplie plus encore les mécontents.

Ces premiers éléments d'analyse concernent une espèce d'ongulé banale. On raisonne donc à partir de densités animales, puis de productivité du milieu, les difficultés de gestion provenant des configurations géographiques de ce dernier. Quand il est peu différencié (cas cévenol), c'est un simple raisonnement trophique (nombre d'animaux sur 100 hectares de châtaigneraie) qui conduit à discuter les rapports sociopolitiques (ici déterminés par des densités relatives d'électeurs) qu'entretiennent le monde de la chasse et celui de l'agriculture. Quand il présente, comme dans les Landes, des couverts végétaux contrastés, le rapport des surfaces en maïs et en pinède constitue une première variable-clé. Elle se complique de la taille unitaire des îlots et parfois de leur rapport périmètre/surface (déterminant pour les coûts et la surveillance des clôtures), qui interdisent à l'agriculteur de savoir si une harde a pénétré sa parcelle et y séjourne ou si elle en est ressortie. Dans le cas des espaces naturels protégés ou des périphéries urbaines, c'est plutôt le rapport entre la population à réguler (et/ou les surfaces à dépeupler) et les effectifs de chasseurs mobilisables qui est en jeu. Ce dernier qualificatif ouvre un débat complexe sur les conditions de recrutement (conditions de sélection, conservation ou non des trophées ou de la venaison, rémunération éventuelle des intervenants. . . ).

\section{Le poids de la réglementation sur le comportement spatial du loup}

Les menaces que les grands prédateurs ont longtemps fait peser sur les hommes et les troupeaux sont à l'origine de leur raréfaction (Baker et al., 2008). Leur survie actuelle dépend donc largement du statut de l'espèce (l'ours et le loup bénéficient de niveaux de protection distincts), mais aussi des conditions de prévention et d'indemnisation des dégâts. Du point de vue géographique, la taille des domaines vitaux augmentant avec celle des espèces, surtout si elles sont carnivores, protéger une population de grands prédateurs par des sanctuaires de taille réduite n'est plus possible. Quand un espace naturel protégé européen couvre quelques centaines de kilomètres carrés, alors que les aires de répartition de l'ours et surtout du loup se chiffrent en dizaines de milliers de kilomètres carrés, on sort d'espaces-milieux sous contrôle étroit d'agents de l'État pour entrer dans les campagnes banales. Les grands ongulés se multipliant, la pratique cynégétique y devient partout présente, tout comme les proies faciles que constituent les animaux d'élevage. De débats à valeur surtout symbolique quand il s'agit d'espaces naturels protégés, les échanges entre habitants favorables et habitants opposés à la présence de ces es- pèces s'adossent alors à des représentations nourries de pratiques.

\section{Les conditions d'installation du loup, puis de sa dispersion}

Les Alpes du Sud affichent les valeurs les plus faibles de l'Hexagone sur une carte des densités humaines; il n'est donc pas surprenant que le loup y ait entamé sa reconquête du territoire français. En Espagne, la répartition de l'espèce (2 000 individus au moins sont recensés) correspond aussi aux déserts des plateaux intérieurs. Dans la sphère "agriculture-élevage », ce sont donc logiquement les productions les plus extensives, adaptées à ces contraintes bioclimatiques, que rencontrent les prédateurs invasifs. Les brebis sont les plus touchées ${ }^{11}$, en particulier les bêtes à viande. Le revenu par tête étant faible, seuls de grands troupeaux (1000 bêtes est un chiffre banal), peu ou pas gardés, procurent aux éleveurs des revenus suffisants. L'apparition des attaques, déstabilisantes, imposa de « redensifier » la part d'humains associée aux troupeaux par des aides-bergers largement financés par la «mesure $\mathrm{t}^{12} »($ Garde, 2007).

À cette vision anthropocentrée, on peut opposer, à une échelle plus fine, celle que le loup porte à son tour sur l'espace. Son comportement alimentaire est organisé à l'aune du rapport entre l'énergie dépensée ou le risque encouru et la ressource obtenue. Lorsque seules des proies sauvages sont disponibles, il prélève les plus accessibles : le mouflon préférentiellement, puis le chevreuil, le cerf, plus rarement le sanglier, voire les petits rongeurs quand ils abondent (Espuno, 2004). Lorsque des proies domestiques sont accessibles, ce sont préférentiellement les ovins, voire les poulains, puis les veaux et enfin les bovins ou chevaux adultes qu'il consomme (Lambert, 2007 ; Poinsot, 2009).

Parmi ces proies potentielles, les premiers loups installés dans le Mercantour disposaient donc d'un large choix. Tant qu'ils étaient peu nombreux, donc que leur densité était faible, les espèces sauvages en hiver puis les animaux domestiques de l'été (moment d'exigences alimentaires accrues, les portées du printemps grandissant) constituaient un régime optimal. Avec les mesures de protection des troupeaux - le triptyque parc de nuit, aides-bergers, chiens de protection (Garde, 2007) -, la mise en espace du prédateur s'est compliquée d'un risque spatialement différencié : certains troupeaux bien placés

\footnotetext{
11 Sur 3133 victimes constatées en 2008, 2963 étaient des ovins (ONCFS, 2009).

12 Définie par une circulaire du ministère de l'Agriculture et de la Pêche en date du 20 septembre 2005 ( «Protection des troupeaux contre la prédation »), cofinancée par l'Union européenne et l'État français, elle prolonge le programme LIFE 1999-2004 intitulé « Le retour du loup dans les Alpes françaises ».
} 
dans le domaine vital devenaient malcommodes à attaquer, tandis que d'autres, moins bien situés par rapport aux remises diurnes ou aux limites territoriales de la meute, étaient moins protégés. L'inégale accessibilité des proies domestiques, d'origine sociale, est donc venue s'ajouter aux différenciations naturelles de l'espace loup. Grâce au statut de protection dont il bénéficiait sur tout le territoire et à l'abondance des proies sauvages et domestiques propres aux espaces-milieux alpins, son aire de répartition s'est étendue à mesure que ses effectifs $\mathrm{s}^{\prime}$ accroissaient ${ }^{13}$.

Les conditions sociales de sa gestion ont alors évolué, les opposants à sa présence se multipliant tandis qu'augmentaient les coûts de la protection-indemnisation des troupeaux ${ }^{14}$. Le statut à la fois écologique et réglementaire de l'espèce a dès lors changé. La population dépassant la centaine d'individus et sa viabilité dans l'Hexagone étant assurée, des tirs de régulation ont été autorisés, modifiant la donne géographique pour les individus ou les meutes. Outre la nécessité d'un domaine vital viable (complémentarité repos-ressources) et d'un territoire suffisant (concurrence intraspécifique), ces tirs constituaient un risque nouveau, spatialement différencié puisque ne pouvant intervenir qu'au-delà $\mathrm{d}^{\prime}$ une certaine fréquence des attaques sur troupeaux ${ }^{15}$. La diffusion spatiale de l'espèce se trouvait ainsi encouragée quand sa densification dans les aires historiques était combattue. Des «zones potentiellement mortelles » se multipliaient dans les régions déjà occupées, alors qu'elles étaient, au moins légalement, absentes des territoires encore vierges. Aux facteurs naturels de dissémination de l'espèce (réduction des coûts énergétiques de capture et concurrence territoriale au sein de l'espèce), s'ajoutaient donc des facteurs sociaux, issus d'une réglementation différenciant spatialement le risque de prédation par les régulateurs officiels.

\footnotetext{
${ }^{13}$ La distance moyenne de dispersion est évaluée en Scandinavie à 313 kilomètres. Cette valeur serait d'autant plus élevée que les densités sont basses, la faible probabilité de rencontrer un individu de sexe opposé incitant aux longs parcours (Wabbakken et al., 2001).

14 On peut ainsi calculer, à partir des chiffres rassemblés par le Centre d'études et de réalisations pastorales Alpes Méditerranée (CERPAM), qu'en 2007, pour une population estimée à 100 individus sur le territoire français, les coûts de protection et d'indemnisation s'élevaient à 30000 euros/animal/an.

15 À partir de 2006, le «protocole technique d'intervention sur les loups » autorise les tirs d'élimination dans les unités pastorales situées en zone de présence permanente (un ou plusieurs loups observés pendant au moins deux hivers) lorsque deux attaques consécutives sont intervenues sur le même troupeau en moins de sept jours. En Valais suisse, les mêmes tirs sont autorisés lorsque plus de vingt-cinq ovins ont été prédatés en moins d'un mois (Romandie News, 12 octobre 2006).
}

\section{En Espagne, les effets collatéraux de la régulation du sanglier}

Une diffusion du loup aux régions faiblement peuplées du territoire français serait attendue, du Massif central (il est installé depuis peu en Cévennes) au Jura et aux Vosges vers le nord comme aux Pyrénées vers le sud. La situation qui règne en Espagne fait cependant douter que ce scénario se réalise complètement. L'animal n'y possède pas le même statut dans toutes les régions. Espèce protégée dans les Asturies, il est chassable en León. Si la population de 2000 individus qui s'y trouve connaît actuellement une extension vers le Sud (Blanco et al., 1992) ${ }^{16}$, aucune progression n'intervient vers l'Ouest, alors que montagnes et troupeaux basques forment a priori un pont de la cordillère cantabrique aux Pyrénées. Les enquêtes réalisées ${ }^{17}$ sur cette barrière révèlent que des battues administratives sont organisées lorsque les dégâts se multiplient, mais le délai de plusieurs jours nécessaire à leur organisation restreint leur efficacité. Rien ne semble donc pouvoir empêcher que le loup s'installe en Pays basque, où les brebis abondent.

Ce n'est qu'à l'examen des pratiques cynégétiques qu'on comprend que la forte densité de sangliers impose des battues fréquentes auxquelles participent en nombre amis et parents d'éleveurs (donc une densité de chasseurs «proportionnelle » à celle des sangliers). Face aux indemnisations tardives ${ }^{18}$ et souvent impossibles faute de cadavre, rapidement nettoyé par les vautours, les pratiquants révèlent à mots couverts qu'en cherchant à déloger un sanglier, c'est parfois un loup qu'ils voient sortir. Les gardes, de leur propre aveu incapables de «savoir ce qui se passe au long d'une ligne de tireurs postés dans ces terrains escarpés », découvrent donc de temps à autre un squelette de loup « régulé » par les battues aux sangliers.

Le loup cohabitant ici avec une forte densité de grands ongulés chassables, tandis que des liens étroits unissent éleveurs et chasseurs, l'intensité de la "régulation grise » (que la loi assimile évidemment à du braconnage) s'élève en certains lieux (Cortès, 2001). Lorsque les indemnisations sont faibles et les dommages socialement insupportables, elle engendre un «milieu » impossible au loup. Ailleurs, en raison d'un contexte réglementaire et administratif plus favorable (dispositif d'indemnisation mieux adapté), de conditions agricoles plus faciles

\footnotetext{
16 Blanco et al. (2005) montrent sa capacité à franchir les fleuves et les autoroutes en utilisant les artifices d'accessibilité (ponts routiers).

17 Effectuées par nos soins dans les conditions précisées par la note 2.

${ }^{18}$ La population lupine n'étant pas estimée en péril, le montant des indemnisations est bien moindre qu'en France : peu ou pas d'aides aux mesures de protection, une indemnisation des brebis sur la base de leur valeur marchande (100 à 200 euros tout au plus).
} 
(moins d'élevages, tout au moins d'ovins), voire de connivences éleveurs-chasseurs moins étroites, l'intensité de cette régulation officieuse est moindre, ce qui permet le maintien de l'espèce. L'ancienneté de son installation de même que la densité de l'espèce influencent aussi la représentation que lycophiles et lycophobes se font de l'animal. Dans leur discours, gardiens de réserve comme chasseurs traitent en effet l'animal d'une manière assez semblable au renard («quand les dégâts augmentent trop, les chasseurs font ce qu'il faut»), le distinguant ainsi de l'ours - plus rare (60 individus seulement pour toute l'Espagne) et aux dégâts moindres -, apparemment plus estimé et respecté (Bobbé, 2002a).

Le statut légal, mais aussi social, du loup en France évoluera donc vraisemblablement à mesure que sa densité comme l'aire de sa présence permanente vont croître. Le souci d'une réduction des coûts d'indemnisation se dessine; les conditions relatives à son effarouchement, voire à sa régulation, s'assouplissent : son basculement du statut de «quasi-ours » à celui de « gros renard » est apparemment en cours.

\section{Conclusion}

Cette analyse de la répartition des animaux par les combinaisons de variables géographiques débouche sur une définition du milieu centrée sur l'étude de l'accessibilité et des formes, actives surtout à grande échelle, et sur celle des densités, plus prégnantes lorsque l'échelle diminue. Dans la réflexion sur ces dernières, deux dimensions suscitent l'intérêt dans une perspective gestionnaire.

La première concerne la répartition géographiquement différenciée des rapports sociaux qui se nouent autour de l'animal. Si leur complexité résulte de données culturelles largement étudiées (par exemple Mauz, 2002; Claeys-Mekdade, 2002), les situations examinées révèlent que les densités relatives des chasseurs et des agriculteurs-éleveurs jouent un rôle déterminant sur le statut de l'animal. Dans les Cévennes, la nonmécanisation imposée par la pente a fait reculer la population agricole à des niveaux très inférieurs à ceux des chasseurs, et a ainsi favorisé le basculement sociologique dans un "milieu cynégétique ». La production de sangliers et de cerfs a alors pris le pas sur l'agriculture, étouffée sous la pression de dégâts dont la maîtrise n'est politiquement plus soutenable - les électeurs-chasseurs rendant par leur nombre les électeurs-agriculteurs quasiment inaudibles. Lorsqu'à l'inverse, dans la montagne basque espagnole, les conditions écologiques et économiques permettent qu'un élevage ovin dense se maintienne, la proportion éleveurs/chasseurs apparaît plus favorable aux premiers. L'animal n'étant plus « ressource» comme dans les Cévennes, mais facteur de dégâts aux ressources (les troupeaux), les chasseurs tirent leur légitimité de leur fonction régulatrice et maintiennent, sous le prétexte du sanglier et au mépris de la loi, une pression sur le loup qui interdit son installation. Prendre en compte ces proportions agriculteurs/chasseurs (et l'évolution du statut de l'animal sauvage à mesure qu'elles évoluent) dans les orientations régionales de gestion de la faune sauvage et des habitats (ORGFSH) ou dans les dispositifs de protection centrés sur les espaces naturels protégés éclaire d'un jour nouveau échecs et succès. On a ainsi montré qu'autour du mouflon, protégé par la Réserve nationale de chasse et de faune sauvage du Caroux-Espinouse, le sud de l'aire de répartition, où vivent de nombreux viticulteurs, voit intervenir une régulation stricte de l'espèce empêchant toute expansion. Au nord et à l'ouest, en des biotopes plus montagnards, l'abondance du sanglier offre une ressource cynégétique aux chasseurs locaux. Elle permet une expansion progressive du mouflon, que contrôle une gestion par des tirs sélectifs effectuant de subtils rééquilibrages par sexes et classes d'âge (Poinsot et Michel, 2009).

La seconde dimension concerne la transformation du milieu géographique qui advient quand la densité d'une espèce évolue. Lorsque les individus sont rares, l'espèce est d'abord patrimoine, donc souvent protégée. Les ressources alimentaires sont alors abondantes, d'éventuelles prédations ou déprédations sont occasionnelles ou dispersées, et la pression sociale et/ou cynégétique est donc très faible. Ainsi, lorsque l'abondance des sangliers était moindre, les dégâts étaient rares, les plans de chasse absents et les chasseurs attirés par l'espèce étaient moins nombreux car les tableaux des battues, inférieurs. De même, pour le loup en phase de colonisation, les individus isolés en quête de partenaire reproductif se déplacent de manière erratique, prédatent majoritairement des proies sauvages et sont donc «invisibles » aux humains.

Lorsque les densités augmentent, qu'un statut de protection concerne l'espèce ou l'espace, ou qu'un milieu nutritionnellement favorable les soutienne (les Cévennes ou les Landes pour le sanglier ${ }^{19}$, les régions d'élevage ovin extensif pour le loup), le milieu de l'animal se transforme. Les tensions sociales qu'exaspèrent les dégâts exigent une pression cynégétique accrue. La fonction «refuge» des réserves réglementaires (ou «de fait » pour les périphéries urbaines) devenant cruciale, les dégâts périphériques se multiplient selon une densité et des formes largement liées à celles des réserves : 1000 hectares d'un seul tenant n'engendreront pas du tout les mêmes dégâts que $10 \times 100$ hectares.

La question des lieux de la pression cynégétique devient alors centrale, toute surpression dans les zones à forte densité d'animaux et de dégâts favorisant les

\footnotetext{
${ }^{19}$ Voire toutes les régions quand se multiplient les agrainages intensifs que pratiquent les chasseurs pour cantonner l'espèce hors des cultures, mais aussi accroître ce qui est devenu une ressource.
} 
dispersions et donc les colonisations « refuges » (du loup hors des zones de présence permanente, des sangliers dans les espaces naturels protégés ou les périphéries urbaines peu chassables). D'un point de vue gestionnaire, la stratégie mise en œuvre en Espagne dans les zones de fortes densités lupines apparaît diamétralement opposée à celle pratiquée en France. Le tir sur les loups est systématiquement encouragé en périphérie de la réserve de la Culebra (Poinsot et Saldaqui, 2012) pour rendre les aires agricoles et d'élevage répulsives à l'espèce, tandis que l'on préserve totalement sa quiétude dans la réserve (au prix d'une aide à la protection plus forte aux éleveurs concernés) afin de favoriser une prédation sur l'ensemble des espèces sauvages, y compris sur les sangliers. Régulés par le loup, ceux-ci occasionnent des dégâts périphériques moindres.

Il paraît enfin utile de revisiter l'interprétation qui est faite du passage des statuts d' $\mathrm{d}^{\prime}$ utile » ou de " nuisible», que mobilisent agriculteurs et chasseurs, à la notion de " partie d'une pyramide trophique à laquelle il est indispensable » qu'emploient les naturalistes. Le regard qu'on porte sur les espèces dépend en effet largement des densités. La rareté justifie la protection et la mise en exergue du rôle dans les chaînes trophiques ou la biodiversité. La forte densité engendre au contraire une qualification dans le registre "ressource », voire une dépréciation dans le registre «nuisible» (qui occasionne des dégâts aux productions ou aux milieux). Penser la gestion des espèces dans une perspective souple, dans le temps et l'espace, adaptant le statut réglementaire aux densités apparaît souhaitable. Comme le prévoit la loi française, et sous réserve $d$ 'harmonisation européenne, départementaliser de manière plus nette le statut des espèces, ainsi que le permettent partiellement les arrêtés préfectoraux relatifs aux régulations du loup ou aux battues administratives sur sangliers, constituerait une solution. Élargissant l'examen au champ de l'avifaune, on pourrait ainsi susciter de manière souple des régulations du cormoran ou du vautour fauve lorsque leurs densités font problème et, à l'inverse, interdir la chasse au grand tétras dans les lieux et temps où sa densité s'effondre. Conditions techniques (Boussin, 2006) et légitimité sociale de l'évaluateur des densités (Saldaqui, 2008) deviennent alors des éléments centraux du débat.

\section{Références}

Arnauduc, J.-P., 2008. Les schémas départementaux de gestion cynégétique au prisme du territoire et du développement durable. Communication au colloque Chasse, territoires et développement durable : outils d'analyse, enjeux et perspectives, organisé par la Société française d'économie rurale, 25-26 mars, ENITAC, Clermont-Ferrand (http:// www.sfer.asso.fr/media/files/colloque_chasse_territoires_ et_developpement_durable_outils_d_analyse_enjeux_et_ perspectives).
Baker, P.J., Boitani, L., Harris, S., Saunder, G., Whites, P.C.L., 2008. Terrestrial carnivores and human food production: Impact and management, Mammal Review, 38, 123-166.

Baubet, E., 1998. Biologie du sanglier en montagne : biodémographie, occupation de l'espace et régime alimentaire. Thèse en biologie des populations, Université Lyon 1, Lyon.

Benhammou, F., 2007. Crier au loup pour avoir la peau de l'ours: une géopolitique locale de l'environnement à travers la gestion de la conservation des grands prédateurs en France. Thèse en sciences de l'environnement et géographie, ENGREF, Paris.

Blanco, J.C., Reig, S., de la Cuesta, L., 1992. Distribution, status and conservation problems of the wolf Canis lupus in Spain, Biological Conservation, 60, 2, 73-80.

Blanco, J.C., Cortes, Y., Virgos, E., 2005. Wolf response to two kinds of barriers in an agricultural habitat in Spain, Canadian Journal of Zoology, 83, 312-323.

Bobbé, S., 2002a. L'Ours et le loup : essai d'anthropologie symbolique, Paris, INRA/MSH.

Bobbé, S., 2002b. Polémique autour du projet de zonage appliqué à la gestion des loups dans l'arc alpin français, Espaces et sociétés, 110-111, 111-128.

Boussin, L., 2006. Société et animal : contrôler la grande faune sauvage. L'exemple du chevreuil dans le département de la HauteVienne. Thèse de géographie, Université de Limoges.

Burel, F., Baudry, J., 1999. Écologie du paysage : concepts, méthodes et applications, Paris, Tec \& Doc.

Claeys-Mekdade, C., 2002. Les controverses relatives à la démoustication de la Camargue : rapports à l'animal et au territoire, Espaces et sociétés, 110-111, 147-165.

Cortes, Y., 2001. Ecologia y conservacion del lobo (Canis lupus) en medios agricolas. PhD Dissertation, University Complutense of Madrid.

Delort, R., 1984. Les Animaux ont une histoire, Paris, Le Seuil.

Espuno, N., 2004. Impact du loup sur les ongulés sauvages et domestiques dans le massif du Mercantour. Thèse de biologie des population et écologie, Université Montpellier 2, Montpellier.

Garde, L. (Ed.), 2007. Loup, élevage : s'ouvrir à la complexité. Actes du séminaire technique des 15 et 16 juin 2006, Aix-en-Provence, Manosque, CERPAM.

Gompper, M.E., 2002. The Ecology of Northeast Coyotes: Current Knowledge and Priorities for Future Research. Working Paper No. 17, Wildlife Conservation Society, Bronx (NY).

Jobin, A., Molinari, P., Breitenmoser, U., 2000. Prey spectrum, prey preference and consumption rates of Eurasian lynx in the Swiss Jura Mountains, Acta Theriologica, 45, 243-252.

Klein, F., Guibert, B., Baubet, E. (Eds), 2008. Modalités de gestion du sanglier: actes du colloque de Reims des $1^{\text {er }}$ et 2 mars 2007, Paris, FNC-ONCFS (+ CD-ROM).

Lambert, B., 2007. La prédation sur troupeaux allaitants : état des lieux des connaissances et de leurs conséquences, in Garde, L. (Ed.), Loup, élevage : s'ouvrir à la complexité Actes du séminaire technique des 15 et 16 juin 2006, Aix-en-Provence, Manosque, CERPAM, 228-239.

Lopez Ontiveros, A., 1991. Geografía de la caza en España, Agricultura y Sociedad, 58, 81-112.

Mauz, I., 2002. La conception de la juste place des animaux dans les Alpes françaises, Espaces et sociétés, 110-111, 129-145.

Mauz, I., 2005. Gens, cornes et crocs, Antony, CEMAGREF / Paris, CIRAD / Versailles, INRA.

Mounet, C., 2007. Les Territoires de l'imprévisible : conflits, controverses et «vivre-ensemble " autour de la gestion de la faune sauvage. Le cas du loup et du sanglier dans les Alpes françaises. Thèse de géographie, Université Grenoble 1, Grenoble. 
ONCFS, 2009. Bulletin d'information du réseau Loup, 20.

Pelosse, V., 1993. Construction de l'animal cynégétique : mouflons et sangliers en Languedoc, Études rurales, 129-130, 73-82.

Planhol, X. de, 2004. Le Paysage animal : l'homme et la grande faune, une zoogéographie historique, Paris, Fayard.

Poinsot, Y., 2008. Les enjeux géographiques d'une gestion durable de la faune sauvage en France, Annales de géographie, 663, 26-47.

Poinsot, Y., 2009. Protection de la grande faune et territoires : deux modèles de gestion dans la cordillère Cantabrique, L'Espace géographique, 4, 289-302.

Poinsot, Y., Michel, P., 2009. La gestion durable des espèces protégées dans leurs territoires : succès et tensions autour du mouflon dans le massif du Caroux-Espinouse, Sud-Ouest européen, 27, 109-118.

Poinsot, Y., Saldaqui, F., 2009. Quelle organisation territoriale pour une gestion durable des sangliers? Un exemple pyrénéen, Mappemonde, 94, 2 (http://mappemonde.mgm.fr/ num22/index.html).

Poinsot, Y., Saldaqui, F., 2012. La maîtrise des populations de grands ongulés dans les espaces naturels protégés : comment gérer la spatialité animale par des territoires humains?, Cybergeo : European Journal of Geography : http:// cybergeo.revues.org/25226 (doi : 10.4000/cybergeo.25226).

Reçu le 22 mars 2010. Accepté le 9 septembre 2011.
Ripple, W.J., Beschta, R.L., 2004. Wolves and the ecology of fear: Can predation risk structure ecosystems?, BioScience, 54, 8, 755-766.

Saldaqui, F., 2008. Le rôle de l'expertise locale dans la gestion concertée de la faune sauvage : l'exemple des guides de chasse de l'Office national des forêts dans leur voisinage. Communication au colloque Chasse, territoires et développement durable : outils d'analyse, enjeux et perspectives, organisé par la Société française d'économie rurale, 25-26 mars, ENITAC, Clermont-Ferrand (http:// www.sfer.asso.fr/media/files/colloque_chasse_territoires_ et_developpement_durable_outils_d_analyse_enjeux_et_ perspectives).

Saniga, M., 2002. Nest loss and chick mortality in capercaillie (Tetrao urogallus) and hazel grouse (Bonasa bonasia) in West Carpathians, Folia Zoologica, 51, 3, 205-214.

Wabakken, P., Sand, H., Liberg, O., Bjärvall, A., 2001. The recovery, distribution, and population dynamics of wolves on the Scandinavian peninsula, 1978-1998, Canadian Journal of Zoology, 79, 710-725.

Waguet, P., Charlez-Coursault, A., 1991. La Chasse en France, Paris, PUF. 